\title{
Assessment of diversity by using morphological, biochemical and molecular approaches of selected basmati rice (Oryza sativa $L$.) varieties
}

\author{
A. Mishra ${ }^{1 *}$ Pradeep Kumar ${ }^{1}$, R. S. Sengar ${ }^{1}$, P. Kumar ${ }^{1}$, R. Singh ${ }^{2}$, R. Chaudhary ${ }^{1}$, \\ A. Kumar ${ }^{1}$, and P. Fatima ${ }^{1}$ \\ ${ }^{1}$ Department of Agricultural Biotechnology Sardar Vallabhbhai Patel University of Agriculture and Technology \\ Meerut- 250110 (U.P.), INDIA \\ ${ }^{2}$ Zonal Research Station, Nagina, Sardar Vallabhbhai Patel University of Agriculture and Technology Meerut \\ 250110 (U.P.), INDIA \\ *Corresponding author. E-mail: anuragmishraa@hotmail.com
}

Received: March 29, 2015; Revised received: October 19, 2015; Accepted: January 24, 2016

\begin{abstract}
The study investigates the genetic diversity among the Basmati rice genotypes. Selected nine Basmati rice genotypes were studied for twelve morphological traits, biochemical parameters and for molecular analysis with 11 SSR markers. Pusa Sugandha 5 and Basmati 370, showed strong aroma while other varieties showed medium aroma. Alkali spreading value were intermediate in Basmati 386, Vallabh Basmati 22 and Vallabh Basmati 24 while other varieties showed high values. Pusa Basmati 1 and Basmati 386 showed soft category of gel consistency while in rest varieties it was under medium category. Amylose percentage in grains were ranged from 18.02\% (Taraori basmati) to $22.0 \%$ (Basmati370). Molecular analysis with 11 SSR markers showed 125 allels with an average number of allels 11.36 per locus. All the markers showed specific type of banding pattern along with 82 polymorphic allels in different genotypes. This study focuses on application of statistical methods and techniques in analysis of genetic diversity of the agronomic data, biochemical aspects related to quality parameters and at the molecular level using SSR markers for clustering procedure making dendrogram that helps the more accurate selection of the superior basmati genotypes for the further studies of the breeders and researchers.
\end{abstract}

Keywords: Amylose, Aroma, ASV Basmati rice, Diversity, GC and SSR Primers

\section{INTRODUCTION}

Basmati rice is premium rice with high aroma and grain quality. After cooking Basmati rice length increases more than twice of its original length. The aroma and its sweet taste make it delicious. Grain and cooking quality analysis of Basmati rice reveals large variation in grain dimensions, brown rice colour, texture of cooked rice, intensity and kind of aroma and adaptation to certain agro-climatic conditions. Grains with similar amylose content, gel consistency and alkali spreading value also show variable cooking behaviours. The molecular characterization is a necessary step to determine whether any germplasm is a part of the gene pool of the system concerned. This is especially important in cases where morphological markers can prove to be inaccurate. Molecular markers like RAPD, SSR and ISSR have been used for the genetic diversity in different crops. These markers are extremely sensitive and are capable of identifying allelic variability in early stage of plant growth. SSR (Simple Sequence Repeat) markers or microsatellites are tandem repeats interspersed throughout the genome and can be amplified using primers that flank these regions (Grist et al., 1993). The assessment of diversity of In- dian rice genotypes has been carried out by many researchers in the past (Saini et al., 2004; Ram et al., 2007; Sundaram et al., 2008, Kumar et al., 2010; Sivaranjani et al., 2010 and Vanniarajan et al., 2012). There are few reports of genetic diversity analysis of basmati rice of Indian continent (Nagaraju et al., 2002). We planned this study to work out the diversity assessment of popular basmati rice varieties of India.

\section{MATERIALS AND METHODS}

The Basmati rice varieties were grown in season 2012 to 2013 at the Crop Research Centre and other laboratory works were conducted at, department of Agriculture Biotechnology, Sardar Vallabhbhai Patel University of Agriculture \& Technology, Meerut (U.P.) at latitude of $29^{\circ} 40^{\prime}$ North and longitude of $77^{\circ} 42^{\prime}$ East and at an altitude of 237 meter above mean sea level.

Evaluation of agronomic trait: Data were recorded on five competitive plants per genotype from the middle row for yield and yield components such as days to flower (DF), days to maturity (DM), plant height (PLH), number of tillers per plant (TLN), panicle length (PNL), panicle bearing tillers per plant (PBT), spikelet fertility percentage (SFP), leaf area (LA), Test 
weight (TW),Biological yield (BY), Harvest index (HI) and Grain yield per plant (GYP).

Evaluation of biochemical traits (quality traits)

Aroma test : Take $5 \mathrm{gm}$ of rice soaked in $15 \mathrm{ml}$ of water for $10 \mathrm{~min}$ and cook for $15 \mathrm{~min}$. Cooked rice transferred into a petri dish and placed in refrigerator for $20 \mathrm{~min}$ for cooling. The cooked rice was evaluated by olfactory method with as, a random panel; strongly scented (SS); mild scented (MS); non scented (NS) (Anonymous, 2004).

Alkali spreading value (ASV) and Clearing test: Six milled rice were taken in Petri plates and $10 \mathrm{ml}$ of potassium hydroxide $(0.34 \mathrm{M} \mathrm{KOH})$ was added to the samples, Samples were kept for 23 hours in an incubator at $27-30{ }^{\circ} \mathrm{C}$ (Perez and Juliano, 1978)

Amylose content (AC): Take $100 \mathrm{mg}$ sample of rice flour $1 \mathrm{ml}$ of $95 \%$ ethanol and $9 \mathrm{ml}$ of $1.0 \mathrm{~N} \mathrm{NaOH}$ were mixed and heated in a boiling water-bath for 10 min. Samples were diluted to $100 \mathrm{ml}$ with distilled water. Take $5 \mathrm{ml}$ diluted sample and $1 \mathrm{ml}$ of acetic acid (57.75 $\mathrm{ml}$ Glacial acetic acid in one litre water) was added to acidify the sample along with $1.5 \mathrm{ml}$ of iodine solution ( $0.2 \%$ iodine $+2 \%$ potassium iodide).Sample of volume was made upto $100 \mathrm{ml}$ with distilled water. The samples were incubated at room temperature for $20 \mathrm{~min}$. The absorbance was measured at $620 \mathrm{~nm}$ using spectrophotometer. As a control, $\mathrm{NaOH}$ solution were used. The AC of different varieties was calculated in comparison with standard graph (Perez and Juliano. 1978).

Gel consistency (GC): Take $100 \mathrm{mg}$ sample of rice flour in test tube $(2 \times 19.5 \mathrm{~cm})$, add $0.2 \mathrm{ml}$ of ethanol containing $0.25 \%$ thymol blue and $2.0 \mathrm{ml}$ of $0.2 \mathrm{~N}$ of $\mathrm{KOH}$ were added and kept in boiling water-bath for 8 min. Cooled, mixed well and kept in ice bath for 20 min. Later the test tubes were laid horizontally for one hour and measurements were made using graph paper. The degree of disintegration of kernel was evaluated using a 7 point scale (Bhattacharya. 1979).

Selection of SSR markers: A prior information was used to select random as well as trait-linked SSR markers. Randomly selected 11 markers having wide genome distribution that could effectively illustrate the diversity among the genotypes, developed by the Cornell University, Ithaca (Mc Couch et al., 2002).

Marker assay: Total genomic DNA isolation was carried out by a CTAB method (Murray and Thompson. 1980) and assayed with a total of 11 SSR markers. The PCR reactions were carried out in sterile $0.2 \mathrm{ml}$ thin-walled PCR tubes. Amplification was carried out in $20 \mu \mathrm{l}$ reaction volume (25-50 ng DNA template $1 \mu \mathrm{l}$, $10 \mathrm{Mm}$ dNTP mixture $0.6 \mu \mathrm{l}, 1 \mathrm{U} / \mu \mathrm{l}$ Taq DNA polymerase $0.5 \mu \mathrm{l}$. PCR Reaction buffer $2.0 \mu \mathrm{l}$, Primer F $1.0 \mu$ l, Primer R $1.0 \mu$ l, Double Distilled water13.5 $\mu$ l. The PCR amplification was done in a thermo-cycler which was programmed as follow denatured at $94^{\circ} \mathrm{C}$ for 3 minutes followed by 35 cycles $(1 \mathrm{~min}$ denaturation at $94^{\circ} \mathrm{C}, 1$ minutes annealing at $55^{\circ} \mathrm{C}$ and $1 \mathrm{~min}$ of primer extension at $72^{\circ} \mathrm{C}$ ) of PCR amplification followed by final extension of $72^{\circ} \mathrm{C}$ for 7 minutes and
$4^{0} \mathrm{C}$ for forever Storage. The amplification products were separated on $2 \%$ agarose gels and run for $2 \mathrm{~h}$ in $1 \mathrm{X}$ TAE buffer. DNA fragments were visualized under UV transilluminator using Alpha Imager gel doc.

Data analysis: The phenotypic data were subjected to statistical analysis for descriptive purposes, as well as for character association. ANOVA analysis using software OPSTAT. The dendrogram was constructed using the software package NTSYSpc (Numerical Taxonomy System). Amplicons were scored for their product size for each primer genotype combination. The informativeness of the markers in terms of polymorphism was determined by calculating the polymorphism information content (PIC) (Botstein et al., 1980) for each SSR locus, according to the formula, $\mathrm{PIC}=1-\sum \mathrm{pi}^{2}$

Where pi is the frequency of the $i$ th allele (Smith et al., 1997)

\section{RESULTS AND DISCUSSION}

Agronomic traits: Morphological traits such as DF, DM, PLH, PL, TLN, PBT, SFP, LA, BY, TW, HI and GYP under study showed wide range of variation in different rice genotypes. Flowering date (DF) ranged from lowest for Basmati 370 (97.38 days) to highest for Type3 (130.05 days). Days of maturity (DM) was recorded minimum for Pusa Sugandha 4(120 days) to maximum for Basmati 386 (156.33 days). The plant height (PLH) ranged from minimum for Pusa Basmati $1(85.68 \mathrm{~cm})$ and maximun for Basmati 370 (153.23 $\mathrm{cm})$. Plant height are highly diverse in different rice varieties (Tahir et al., 2002 and Golam et al., 2011).Tillers numbers per plant (TLN) was recorded minimum for Pusa Sugandha 5 (10.40 tillers) to maximum for Basmati 370 (20.13 tillers) tillers per plant.Panicle bearing tillers per plant (PBT) ranged from Pusa sugandha 5 for minimum is 8.69to maximum for Basmati 370 is18, The panicle length (PNL) of plant ranged from minimum isPusa sugandha 4 is $28.41 \mathrm{~cm}$ to maximum for Basmati 370 is $34.18 \mathrm{~cm}$. the spikelet fertility percentage (SFP) is ranged from Taraori Basmati $(94.47 \%)$ to highest for Basmati 370 $(97.70 \%)$.The leaf area (LA) was recorded $35.98 \mathrm{~cm}$ minimum for Vallabh Basmati 22 to $65.68 \mathrm{~cm}$ maximum for Basmati 370. The biological yield (BY) ranged from minimum for ( Pusa Basmati 1 ) to maximum for Basmati 386 (167.66 q/ha). Test weight is ranged from Basmati 370 (21.26 gm), to Pusa sugandha 4 (28.43 gm). The grain yield (GY) ranged from minimum for Type $3(33.32 \mathrm{q} / \mathrm{ha})$ to maximum for Vallabh Basmati 24 (64.21 q/ham). The Harvest index (HI) was observed minimum for Type 3 (23.32 $\%)$ to maximum for Vallabh Basmati 24 (55.25\%). The mean performances of nine genotypes for twelve characters are given in Table1. All the varieties showed the different pattern in morphological characters.

\section{Biochemical Traits (Quality traits)}

Aroma test: Basmati 370 and Pusa Sugandha 5 showed the presence of strong aroma. Type 3, Pusa 


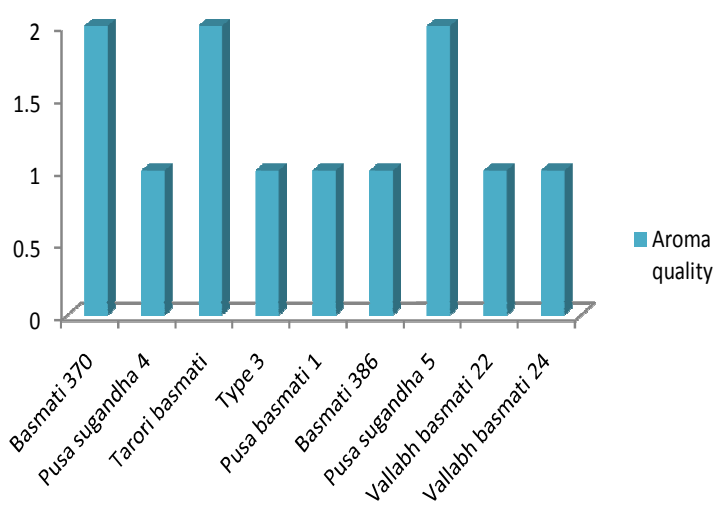

Fig.1. Aroma test for nine selected rice varieties.

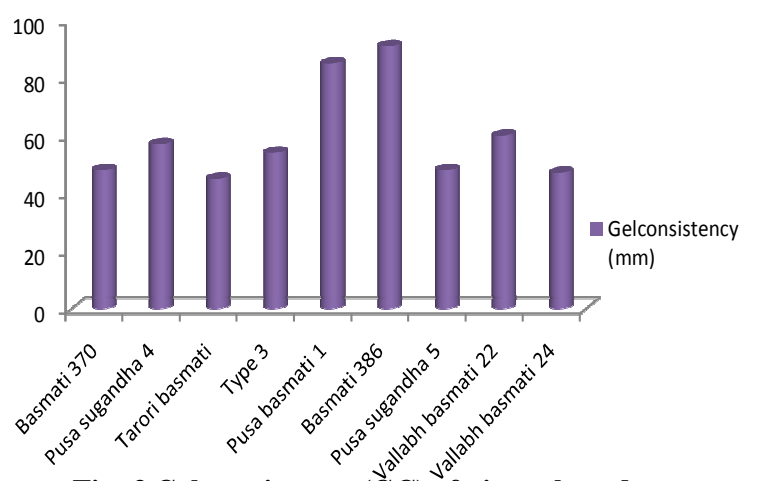

Fig. 3. Gel consistency $(G C)$ of nine selected rice varieties.

Basmati 1, Basmati 386, Vallabh Basmati 22 and Vallabh Basmati 24 showed presence of mild aroma (Fig.1). Pusa Basmati 1 and Pusa sugandha 5 contains more aroma than other traditionally cultivated scented rice varieties (Nadaf et al., 2006).

Alkali Spreading Value and Gelatinization: Alkali spreading value were recorded as high, intermediate and low. Alkali Spreading Value (ASV) and Gelatinization test (GT) (Table2, Fig. 2) revealed that low ASV (1-3) and high GT were detected in Type 3, Basmati 370, Pusa Sugandha 4, Taraori Basmati, Pusa Basmati 1, and Pusa Sugandha 5. The intermediate alkali spreading value and gelatinization were observed in basmati 386, Vallabh Basmati 22, and Vallabh Basmati 24 varieties. The traditional rice varieties shows low ASV and high gelatinization temperature which is highly desirable for the grain quality (Bansal et al., 2006). Gelatinization is the process in which the intermolecular bonds of starch molecules are broken down in the presence of water and heat, thereby dissolving the starch granules in the endosperm. The gelatinization temperature determines the time required for cooking.

Gel consistency: Gel consistency (GC) were measured as soft, medium, and hard among basmati rice varieties. Gel consistency ranged from 65 to $100 \mathrm{~mm}$ as soft, 41 to $64 \mathrm{~mm}$ as medium and 26 to $40 \mathrm{~mm}$ hard.

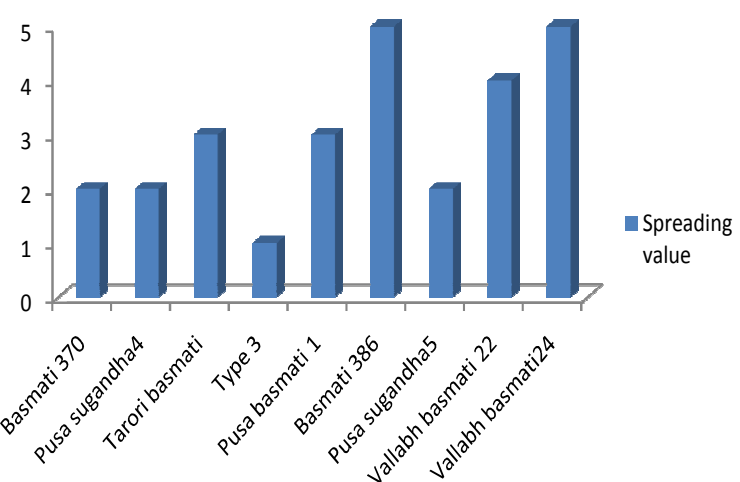

Fig. 2. Alkali spreading value (ASV) of nine selected rice varieties.

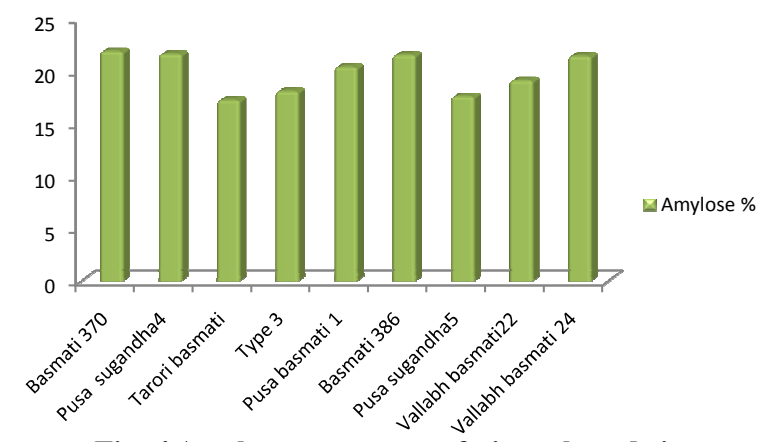

Fig. 4. Amylose percentage of nine elected varieties.

The length of the blue gel was maximum $(91 \mathrm{~mm})$ in Basmati 386 and fallowed by $(85 \mathrm{~mm})$ Pusa Basmati. The varieties Pusa Sugandha 5, Vallabh Basmati and Vallabh Basmati 24 recorded $48 \mathrm{~mm}, 22 \mathrm{~mm}, 60 \mathrm{~mm}$ and $47 \mathrm{~mm}$ length of blue gel respectively and categorized under medium gel consistency. The lowest GC was recorded in Taraori Basmati $(45 \mathrm{~mm})$ (Table 3, Fig.3). Gel consistency refers as a parameter to index the tendency of cooked rice under soft to hard on cooling and is normally classified as hard, medium, and soft (Chen et al., 2012).

Amylose content: Rice varieties are grouped on the basis of their AC into waxy (0-2\%), very low (3-9\%), low (10-19\%), intermediate (20-25\%), and high (>25\%) (Shahidullah et al., 2009). Low amylase content was observed in Taroari Basmati (18.02\%), Type $3(18.57 \%)$ and Pusa Sugandha $5(18.86 \%)$. The amylose content was under intermediate range in 19.80\% Vallabh Basmati22 to 22.0\% (Basmati 370). High amylase conternt was recorded in Basmati 370 (22.00\%), followed by Pusa Sugandha 4 (21.40\%), Pusa basmati $1(20.56 \%)$, Basmati $386(21.70 \%)$ and Vallabh Basmati 24 (21.64\%) (Fig.4). Most of the traditional aromatic varieties showed low amylose content in comparison with evolved Basmati rice varieties. Cooking quality is mainly determined by the content and composition of starch (amylose and amy- 

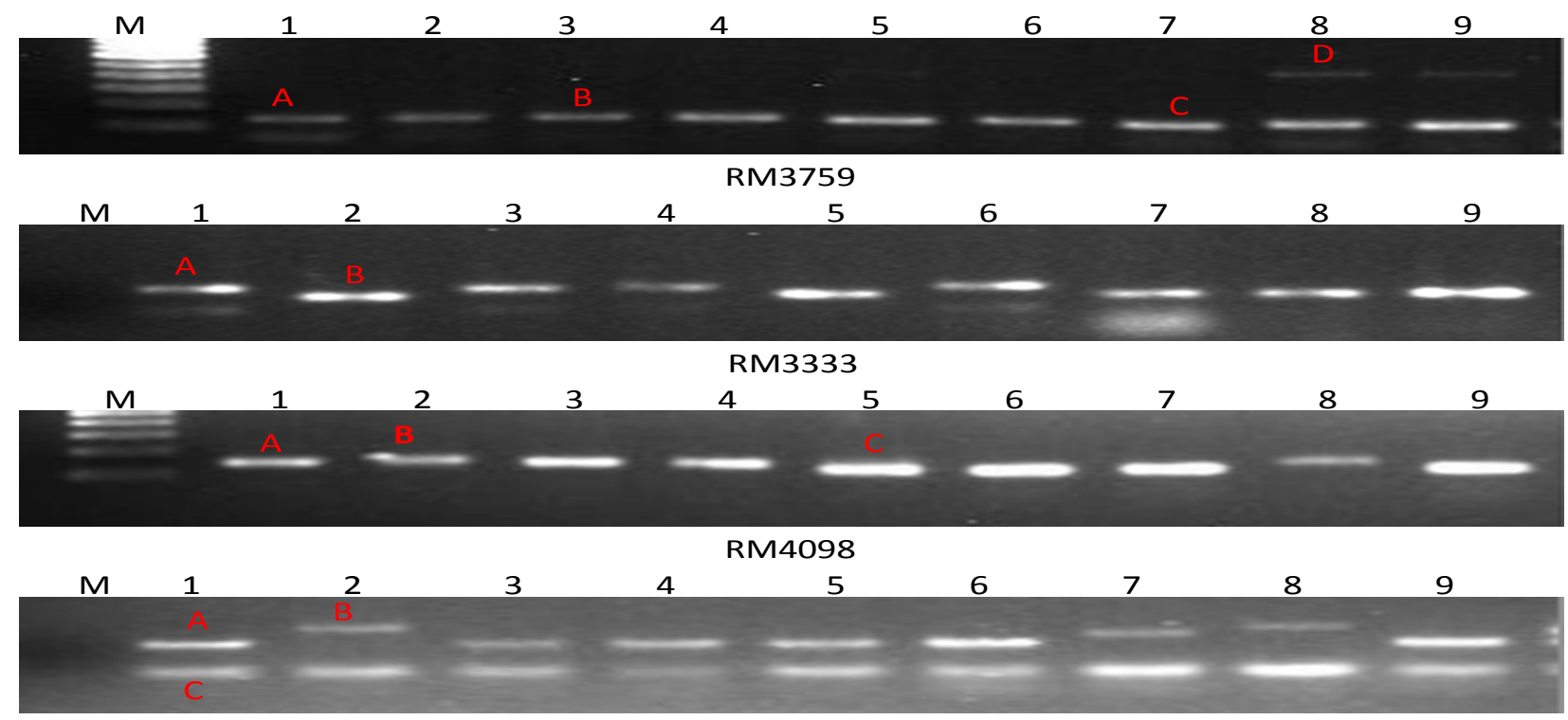

RM1067

Fig. 5. A representative gel picture showing allelic diversity for different SSR markers; $M$, 100-bp ladder; 1-9, rice germplasm lines; A, B, C, and D, different alleles amplified.

lopectin) and storage protein in the endosperm are the main factors (He and Suzuki. 1987; Sowbhagya et al., 1987; Rani and Bhattacharya. 1989; Ong and Blanshard. 1995; Singh et al., 2003; Cameron and Wang 2005; Allahgholipour et al., 2006; Kibanda and Luzikihupi. 2007). Amylose content and the chain structure of amylopectin were found to be significantly correlated with hardiness and stickiness of the rice after cooking (Ramesh et al., 1999; Mizukami and Takeda 2000; Nakamura and Juliano. 2006). The amylose content in all grades of rice ranges between $20.7-21.4 \%$. After cooking rice become moist and sticky due to low amylase content (Shahidullah et al., 2009). High amy- lose grains cook dry, are less tender, and become hard upon cooling, In contrast, low-amylose rice cooks moist and sticky. Intermediate amylose rice is preferred in most rice growing areas of the world (Randall et. al., 2009).

Molecular analysis: DNA primers have the ability to differentiate different genotype based on the difference in the representing genomic regions and also depend upon the number of alleles. Out of eleven, six SSR markers were found to be polymorphic (Fig. 5). The average PIC of these markers was 0.36exhibiting the highest PIC 0.74 . The average number of polymorphic alleles is 3.67 , amplified by SSR Primers led to the

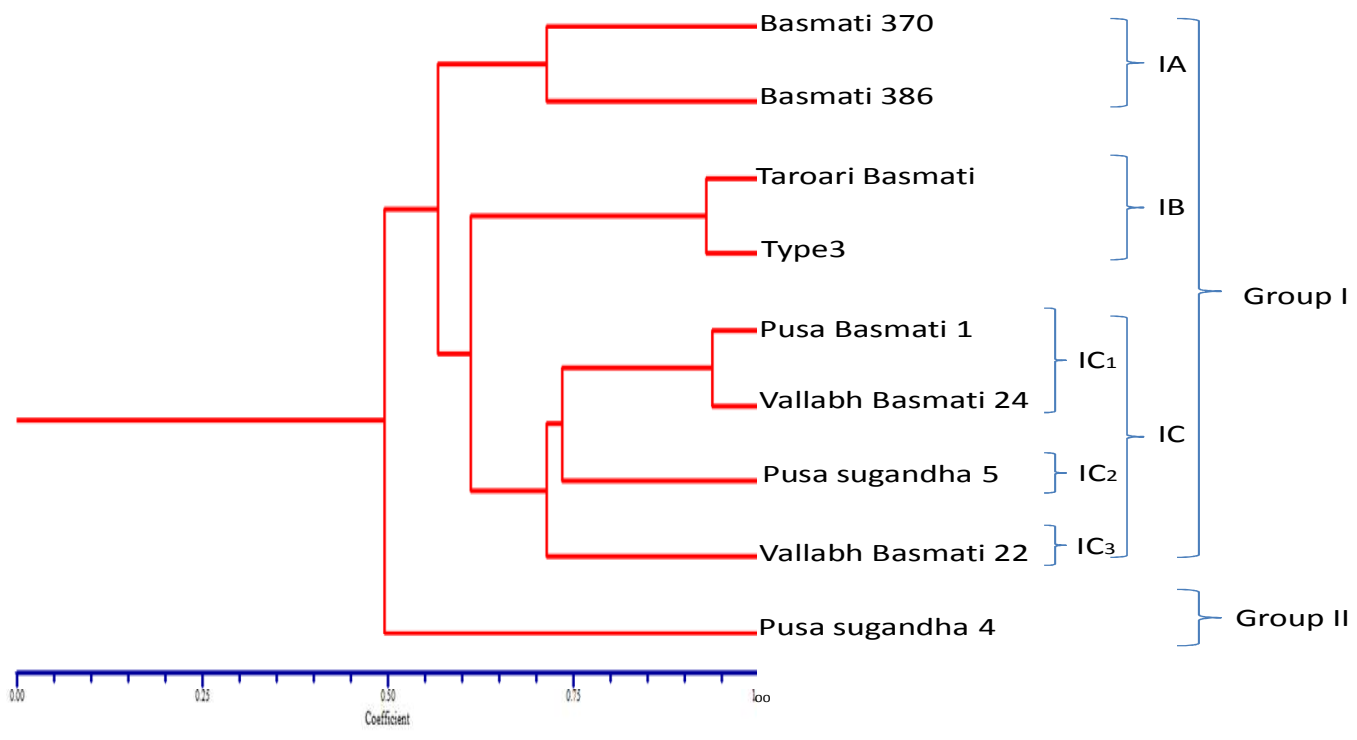

Fig.6. Moleculardendrogram showing the genetic similarity among aromatic rice varieties. 


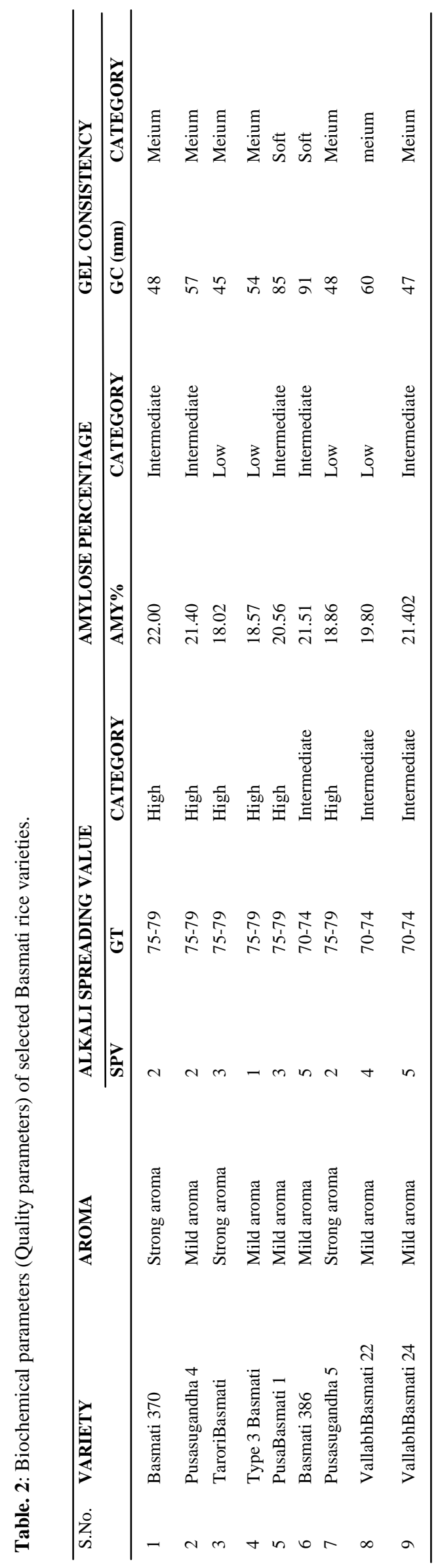

amplification of total 125 bands, out of which 82 bands are polymorphic and while 43 bands were monomorphic. Primer RM 4469 and RM 1067 amplify maximum number of bands andRM4405 and RM1003 amplified minimum number of bands. On an average 11.36 bands amplified per primer. The primers pair RM4469 and RM1067 with an average number of PIC value 0.97 is maximum for SSR primer. All nine cultivars are grouped (Fig. 7) into two major groups (Group I and Group II) based upon the Multivariate analysis of genetic similarity data. At the coefficient $\mathrm{J}=0.50$ Group I consist of eight genotype, Basmati 370, Basmati 386, Taroari Basmati, Type 3, Pusa Basmati 1, Vallabh Basmati 24, Pusa sugandha 5 and Vallabh Basmati 22 whereas Group II consist of one genotype, Pusa Sugandha4, Group Iis further divide into three sub groups ( IA , IB and IC). At the coefficient of $\mathrm{J}=$ 0.55 to 0.60 subgroup IA consist of two genotypes, Basmati 370 and Basmati 386 at the coefficient $\mathrm{J}=$ 0.70. Group IB also consist of two genotypes, Taroari Basmati and Type 3 at the Coefficient $\mathrm{J}=0.94$. Whereas subgroup IC further divided in three sub group $\left(\mathrm{IC}_{1}, \mathrm{IC}_{2}\right.$ and $\mathrm{IC}_{3}$ ). Subgroup $\mathrm{IC} 1$ consist of two genotype, Pusa Basmati 1 and Vallabh Basmati 24 at the coefficient $\mathbf{J}=0.94$ and subgroup $\mathrm{IC}_{2}$ consist of one genotype Pusa sugandha 5 at the coefficient $\mathrm{J}=$ 0.74. SubgroupIC $\mathrm{C}_{3}$ also consist of single genotype Vallabh Basmati 22 at the coefficient $\mathrm{J}=0.72$. Highest genetic similarity $(93.75 \%)$ measured between PusaBasmati 1 and Vallabh Basmati 24, Taroari Basmati and Type 3 also closely related (92.85\%), Basmati 370 and Basmati 386 lies in same group but coefficient $\mathrm{J}=$ 0.70 , while least genetic similarity $(38.88 \%$ ) was observed between Pusa sugandha 4 and Taroari Basmati. These phenomenon could be support towards strong bias power of some of the DNA marker. Highest PIC value were observed for SSR primers RM3759 is 0.748 and RM4335 is 0.748 . PIC value is reflection of allele diversity and frequency among the cultivars (Lapitan et al., 2007 and Wang. 2009). The average PIC value for SSR loci was 0.3632. The number of alleles detected by microsatellite marker varied from 2 to 3 with an average of 11.36 alleles per locus, whilesome other workers found an average of 4.5, 14.6, 7.7, 13.0, 4.5 and 4.4 alleles per locus (Jalaluddin et al., 2007, Siwach et al., 2004; Brondani et al., 2006; Oliveira et al., 2007; Thompson et al., 2007, Ghneim et al., 2008 and Pervaiz et al., 2009, 2010). Five markers yielded two alleles while RM3332, RM4405and RM1067 markers produce three bands.

In this study genetic diversity of Basmati rice shows clear group of traditional basmati (Basmati 370, Basmati 386, Taroari Basmati and Type3) and evolved Basmati (Pusa Basmati 1, Pusa sugandha 5, Vallabh Basmati 22 and Vallabh Basmati 24) rice genotypes. The results indicate significant genetic variation among the genotypes. Microsatellite assays identified a number of alleles that were shared by all the Basmati varieties. 
Table. 3: List of SSR (Simple sequence repeats) primers and their sequences.

\begin{tabular}{llll}
\hline S.No. & Primers & Forward Sequences & \multicolumn{1}{c}{ Reverse Sequences } \\
\hline 1 & RM1003 & '5GATTCTTCCTCCCCTTCGTG3' & '5TTCCTGTCAGAACAGGGA3' \\
2 & RM1019 & '5GTTTGAACAGTAGGACTTGT3' & 5AGAACATCTCACACTTCTCT3' \\
3 & RM3332 & '5ATTCTCCTCGCTTCCTCCTC3' & '5AAAGGAGAGAGCCGAGCACTG3' \\
4 & RM3759 & '5GCCACCCACTTTGAGCTTAG3' & 5GATGCTGGTGCGGATCTG3' \\
5 & RM4098 & '5CGTTTGGATGAAGAAGAAGA3' & 5AGTGTTCGTTTCGGATTAGA3' \\
6 & RM4469 & '5AATTTCTCATGTTTTCTTCC3' & 5AGTTATTCTAAGGGAGGGAC3' \\
7 & RM4405 & '5TGAAGCAATTTGATTTTCAG3' & 5GAGCTGGCCTTTATTAACTG3' \\
8 & RM3333 & '5GAAGCAAAAGCTATCGACAC3' & 5GAGCTTGTTGGCACCTTAC3' \\
9 & RM4355 & '5GGGATGAGAGTAGAAGGCA & '5TATATGGCAAGCCTAGCG3' \\
10 & RM4321 & '5AGAAGGAAAGGTGATGATGA3' & 5CCAACGTGAGGTTTATAACA3' \\
11 & RM1067 & '5CGATCCAGAGAGAATGTCTAG3' & '5TAATACGCAAGGCAGAAGGG3' \\
\hline
\end{tabular}

\section{Conclusion}

Traditional varieties Basmati 370, Taroari Basmati is used as donor parent programme for developing new high yielding evolved varieties of aromatic and basmati rice under breeding programmes. In present study morphological characters showed traditional basmati (Basmati 370, Taroari Basmati, Type 3) lies in same group for date of maturity, plant height, number of tillers, panicle bearing tillers, spiklet fertility percentage, while rest of other varieties followed diverse pattern. Biochemical traits study revealed that traditional basmati rice varieties recorded strong aroma, low ASV and intermediate to low AC. Molecular markers analysis revealed close relation among traditional basmati rice varieties. Basmati-370 and Basmati -386 showed very close relation with Taroari Basmati and type-3. The evolved basmati rice varieties also showed very close genetic association. Thus such morphological, biochemical and molecular markers study will be useful for diversity and the more accurate selection and study of superior genotypes.

\section{ACKNOWLEDGEMENT}

The first author is thankful to Dr.H.S.Gaur, Vice Chancelor of the S.V.P.U.A.\&.T. Meerut. Author is also thankful to the Department of Agricultural Biotechnology, S.V.P.U.A. \&.T. Meerut. For providing research facility.

\section{REFERENCES}

Allahgholipour, M.A.J.A., Alinia, F., Nagamine, T., and Kojima, Y. (2006). Rice grain amylase and pasting properties for breeding better quality rice varieties. Plant Breeding, 125:357-362.

Anonymous (2004). Laboratory Manual on Rice Grain Quality Procedure. Directorate of Rice Research, Rajendranagar, Hyderabad, India 1-20.
Bansal, U.K, Kaur, H., \& Saini R. G. (2006). Donors for quality characteristics in aromatic rice. Oryza, 43 (3):197-202.

Bhattacharya, K.R. (1979). Tests for parboiled rice in Proc. Wkshp. Chem. Aspects of Rice Grain Quality. Int. Rice Res. Inst., Los Bahos, Laguna, Philippines p 363.

Botstein, D., White, R.L., Skolnick, M. and Davis, R.W. (1980). Construction of a genetic linkage map in man usingrestriction fragment length polymorphisms. American Journal of Human Genetics, 32:314-331.

Brondani, C., Borba, T.C.O., Rangel. Paulo, H.N. and Brondani, RPV (2006). Determination of genetic variability of traditional varieties of Brazilian rice using microsatellite markers. Genetics and Molecular Biology, 29 (4): 676-684.

Cameron, D.K., and Wang, Y. (2005). A better understanding of factors that affect the hardness and stickiness of longgrain rice. Cereal Chemistry, 82:113-11.

Chen,Y., Wang, M., Ouwerkerk and Pieter, B.F. (2012). Molecular and environmental factors determining grain quality in rice. Food and Energy Security, 1 (2): 111132.

Ghneim, H.T., Duque, P.D., Almeida, I.P., Nunez, T.G., Pieters, A.J., Martinez, C.P. and Tohme, J.M. (2008). Assessment of genetic diversity Molecular Biology and Biotechnology, Indira Gandhi Krishi Vishwavidalaya. Research Journal of Agriculture Sciences,4 (3): 329332.

Golam, F., Yin, Y.H., Masitah, A., Afnierna, N., Majid, N.A., Khali, N. and Osman, M. (2011). Analysis of aroma and yield component of aromatic rice in Malaysian tropical environment. Journal of Crop Science, 5 (11): 1318-1325.

Grist, S.A., Firgaira, F.A. and Morley, A.A. (1993). Dinucleotide repeat polymorphisms isolated by the polymerase chain reaction. Bio-Technology, 15: 304-309.

He, G., and Suzuki, H. (1987). The relationship between translucency of rice grain and gelatinization of starch in the grain during cooking. Journal of Nutritional Science and Vitaminology, 33:263-273.

Jalaluddin, M., Nakai, H. and Yamamoto, T. (2007). Genetic diversity and DNA fingerprinting of some modern in- 
dica and japonica rice. SABRAO Journal of Breeding and Genetics, 39 (1): 43-52.

Kibanda, J.M.N. and Luzi-kihupi, A. (2007). Influence of genetic and genotype environment interaction on quality of rice grain. African Crop Science Journal, 15: 173182.

Kumar, S., Bisht, I.S. and Bhatt, K.V. (2010). Assessment of genetic diversity among rice (Oryza sativa $\mathrm{L}$.) landrace populationsunder traditional production using microsatellite (SSR) markers. Electronic Journal of Plant Breeding, 1: 474-483.

Lapitan, V.C., Brar, D.S., Abe,T .and Redona, E.D. (2007). Assessment of genetic diversity of Philippines rice cultivars carrying good quality traits using SSR markers. Breeding Science, 57: 263-270.

McCouch, S.R., Chen, X. and Cho, Y.G. (2002). Sequence divergence of rice microsatellites in Oryza and other plant species. Molecular Genetics and Genomics, 268, 331-343.

Mizukami, H. and Takeda, Y. (2000). Chewing properties of cooked rice from new characteristic rice cultivars and their relation to starch molecular structures. Journal of Applied Glycoscience, 47:61-65.

Murray, M.G. and Thompson, W.F. (1980). Rapid isolation of high molecular weight plant DNA. Nucleic Acid Research, 8: 432-435.

Nadaf, A.B., Krishnan, S. and Wakte, K.V. (2006). Histochemical and biochemical analysis of major compound (2-acetyl-1-pyrroline) in basmati and other scented rice (Oryza sativa. L.), Current Science, 91: 1533-1536.

Nagaraju, J., Kathirvel, M., Ramesh Kumar, R., Siddiq, E.A., and Hasnain S.E. (2002). Genetic analysis of traditional and evolved Basmati and non-Basmati rice varieties by using fluorescence-based ISSR-PCR and SSR markers. PNAS 99: 5836-5841.

Nakamura,Y., Sato, A. and Juliano, B.O. (2006). Shortchainlength distribution in debranched rice starches differing in gelatinization temperature or cooked rice hardness. Starch/St€arke 58:155-160.

Oliveira, M.M., Martins, P., Jayamani, S. and Negrao, B. (2007). Genetic relatedness of Portuguese rice accessions from diverse origins as assessed by microsatellite markers. Crop Science, 47: 879-884.

Ong, M.H., and Blanshard, J.M.V. (1995). Texture determinants in cooked, parboiled rice. I: Rice starch amylose and the fine structure of amylopectin. Journal of Cereal Science, 21:251-260.

Perez, C.M. and Juliano, B.O. (1978). Modification of the simplified amylose test for milled rice. Starch, 30:424426.

Pervaiz, Z.H., Rabbani, M.A., Pearce, S.R. and Malik, S.A. (2009). Determination of genetic variability of Asian rice (Oryzasativa L.) varieties using microsatellite markers. African Journal of Biotechnology, 8:641-5651.

Pervaiz, ZH., Rabbani, M.A., Khaliq, I., Pearce, S.R. and Malik, S.A. (2010). Genetic diversity associated with agronomic traits using microsatellite markers in Pakistani rice. Electronic Journal of Biotechnology, 13: 4-5.

Ram, S.G., Thiruvengadam, V. and Vinod, K.K. (2007). Genetic diversity among cultivars, landraces and wild relatives of rice as revealed by microsatellite markers. Journal of Applied Genetics, 48: 337-345.
Ramesh, M., Zakiuddin, S.A., and Bhattacharya, K.R. (1999). Structure of rice starch and its relation to cookedrice texture. Carbohydrate Polymer, 38:337-347.

Randall, G., Mutters, James, and Thompson, F., (2009). Rice quality in the global market, Rice quality handbook. Chapter 1:1-22.

Rani, M.R.S., and Bhattacharay, K.R. (1989). Slurry viscosity as a possible indicator of rice quality. Journal Texture Studies, 20:139-149.

Saini,N., Jain, N., Jain, S. and Jain, R.K. (2004). Assessment of genetic diversity within and among basmati and nonbasmati rice varieties using AFLP,ISSR and SSR markers. Euphytica, 140: 133-146.

Shahidullah, S.M., Hanafi, M.M., Ashrafuzzaman, M., Ismail, M.R., and Khair, A. (2009). Genetic diversity in grain quality and nutrition of aromatic rice's. Afrian Journal of Biotechnology, 8 (7): 1238-1246.

Singh. N., Singh. J., Kaur, L., Sodhi, N.S. and Gill, B.S. (2003). Morphological, thermal and rheological properties of starches from different botanical sources. Food Chemistry81: 219-231.

Sivaranjani, A.K.P. (2010). Assessment of genetic diversity among basmati and non-basmati aromatic rices of India using SSR markers. Current Science, 99: 221-226.

Siwach, P., Jain, S., Sain, N., Chowdhury,V.K. and Jain, R.K. (2004). Allelic diversity among Basmati and nonBasmati long-grain indica rice varieties using microsatellite markers. Journal of Plant Biochemistry and Biotechnology, 13: 25-32.

Smith, J.S.C., Chin, E.C.L., Shu, H., Smith, O.S., Wall, S.J., Senior, M.L., Mitchell, S.E., Kresovich, S. and Zeigle J. (1997). An evaluation of the utility of SSR loci as molecular markers in maize (Zea mays L.) comparisons with data from RFLPs and pedigree. Theoretical and Applied Genetics, 95:163-173.

Sowbhagya, C.M., Ramesh, B.S. and Bhattacharya, K.R. (1987). The relationship between cooked-rice texture and the physicochemical characteristics of rice. Journal of Cereal Science, 5:287-297.

Sundaram, R.M., Naveenkumar, B., Biradar, S.K., Balachandran, S.M., Mishra,B. and Ahmed, M.I. (2008). Identification of informative SSR markers capable of distinguishing hybrid rice parental lines and their utilization in seed purity assessment. Euphytica, 163: 215-224.

Tahir, M.H.N., Sadaqat, H.A. and Bashir, S. (2002). Correlation and path co-efficient analysis of morphological traits in sunflower (Helianthus annuus L.) Populations. International Journal of Agricultureand Biology 4 (3): 341-343.

Thompson, M.J., SeptininJih, E.M., Suwardjo, F., Santoso, T.J., Silitonga, T.S. and Mcouch S.R. (2007). Genetic diversity analysis of traditional and improved Indonesian rice (Oryza sativa L.) germplasm using microsatellite markers. Theoretical and Applied Genetics, 114: 559- 568

Vanniarajan, C., Vinod, K.K. and Pereira, A. (2012). Molecular evaluation of geneticdiversity and association studies in rice (Oryza sativa L.). Journal of Genetics, 9: 9-19.

Wang, F. (2009). Integrated KL (K-means-Laplacian) clustering: a new clustering approach by combining attribute data and pairwise relations. In proceeding of SDM 09, SIAM Press, pp. 38-48. 